\title{
Binary pulsars and tests of general relativity
}

\author{
I. H. Stairs \\ Dept. of Physics and Astronomy, University of British Columbia, \\ 6224 Agricultural Road, Vancouver, B. C., V6T 1Z1, Canada, and \\ Centre for Astrophysics \& Supercomputing, Swinburne University of Technology, \\ Mail 39, P. O. Box 218, Hawthorn, Vic 3122, Australia \\ email: stairs@astro.ubc.ca
}

\begin{abstract}
Binary pulsars are a valuable laboratory for gravitational experiments. Doubleneutron-star systems such as the double pulsar provide the most stringent tests of strong-field gravity available to date, while pulsars with white-dwarf companions constrain departures from general relativity based on the difference in gravitational binding energies in the two stars. Future observations may open up entirely new tests of the predictions of general relativity.
\end{abstract}

Keywords. pulsars: general, pulsars:individual (J0737-3039A/B), gravitation

\section{Introduction: Observing and Using Pulsars}

The 1974 discovery of a pulsar in a binary system (Hulse \& Taylor 1975) provided the experimental gravity community with a precision clock embedded in the distorted space near a compact object. The tremendous potential of such a system for testing gravitational theories was quickly recognized (Wagoner 1975; Eardley 1975; Damour \& Ruffini 1974; Barker \& O'Connell 1975). Since then, pulsars have been discovered in similar and in even more relativistic orbits, and many of these binary systems can be used to test aspects of general relativity (GR) and other theories of gravity. This article gives an overview of these tests; see also Stairs (2003) for a fuller though somewhat dated description of much of this material, and Stairs $(2005,2006)$ for slightly updated versions.

Pulsar observing and timing are discussed thoroughly in other sources (e.g., Lorimer \& Kramer; Bailes 2009), but a few relevant points are worth mentioning as background to gravitational tests. Pulsar timing relies on the observed reproducibility of pulse profiles when averaged over timescales of minutes to hours. This allows the determination of pulse Times of Arrival (TOAs) by cross-correlation with a "standard profile" for a given pulsar. TOA precision improves for pulsars with high signal-to-noise ratio and sharp pulse-shape components. Pulsar timing is accomplished by enumerating the pulsar rotations between TOAs and fitting ephemerides that include spin, astrometric and dispersion parameters as well as full descriptions of any binary orbits. Many gravitational tests depend on thorough understanding of pulsar orbits.

By considering a pulsar's spin and spin derivative, estimates can be made of its surface dipolar magnetic field strength as well as its spin-down or "characteristic" age. Most of the pulsars used in relativity tests have been recycled by transfer of mass and angular momentum as their binary companions evolve (e.g., Tauris \& van den Heuvel 2006), and have millisecond spin periods and surface magnetic fields of $10^{8}-10^{9} \mathrm{G}$. A millisecond pulsar (MSP) in a nearly circular orbit typically has a white-dwarf companion, and there is an expectation that both stellar spin vectors will have been aligned with the orbital angular momentum during the mass transfer phase (e.g. Phinney 1992). A pulsar in an 
eccentric orbit is more likely to have a companion that is also a neutron star, such that the last event in the system was a supernova explosion that could have misaligned one or both spins with the post-explosion orbital angular momentum (e.g. Wex et al. 2000).

Pulsars in both types of orbits (and, indeed, some isolated pulsars as well) find uses in gravitational tests. It should be noted that, although the pulsar orbits known to date do not test strong-field gravity in the sense of approaching the event horizon, the use of pulsars and white dwarfs (and someday perhaps black holes) in these tests does mean that the tests must consider the theoretical predictions for objects that are strongly selfgravitating. For example, many of the tests rely on a extension of the definitions of the Parametrized Post-Newtonian (PPN) parameters (Will \& Nordtvedt 1972; Will 2006) to generalized tensor-multiscalar gravitational theories (Damour \& Esposito-Farèse 1992a; Damour \& Esposito-Farèse 1996). In this description, for example, the PPN parameter $\alpha_{1}$ becomes $\hat{\alpha_{1}}=\alpha_{1}+\alpha_{1}^{\prime}\left(c_{1}+c_{2}\right)+\cdots$, where $c_{i}$ describes the "compactness" of mass $m_{i}$. The compactness is $c_{i}=-2 \partial \ln m_{i} / \partial \ln G \simeq-2\left(E^{\text {grav }} /\left(m c^{2}\right)\right)_{i}$, where $G$ is Newton's constant and $E_{i}^{\text {grav }}$ is the gravitational self-energy of mass $m_{i}$. Compactnesses are about -0.2 for a neutron star (NS) and $-10^{-4}$ for a white dwarf (WD).

\section{Equivalence Principle Violations}

Equivalence principles are thoroughly described in, e.g.,Will (1993). General relativity incorporates even the Strong Equivalence Principle, predicting gravitational results independent of self-gravity, but this and other equivalence principles may be violated in alternate theories of gravity.

Pulsar timing sets limits on $\hat{\alpha_{1}}$, which would imply preferred-frame effects by violation of Lorentz invariance, and $\hat{\alpha}_{3}$, which would additionally imply non-conservation of momentum if non-zero. Pulsar can also constrain other SEP-violation effects produced by various combinations of the (modified) PPN parameters: the Nordtvedt effect, dipolar gravitational radiation, and changes in Newton's constant.

\subsection{Orbital Polarization Tests}

Nordtvedt (1968) proposed direct tests of the SEP through Lunar Laser Ranging (LLR) experiments. The principle behind these tests is that the different contributions of selfgravitation to the masses of the Earth and Moon would cause them to accelerate differently in the gravitational field of Sun, resulting in a "polarization" of the orbit in the direction of the Sun. LLR tests have set a limit of $\eta=(4.4 \pm 4.5) \times 10^{-4}$ (Williams et al. 2009), where $\eta$ is a combination of PPN parameters.

Binary pulsar tests of the SEP and of $\hat{\alpha_{1}}$ and $\hat{\alpha_{3}}$ look for the same type of phenomenon: polarization of orbits of pulsar-white-dwarf systems in preferred directions given by the projection of "extra" acceleration vectors onto the planes of the orbits. The prototype of these tests is the Nordtvedt-equivalent SEP test, which considers a strong-field version of $\eta$ labeled $\Delta_{i}$, with pulsar-white dwarf systems constraining the difference $\Delta_{\text {net }}=$ $\Delta_{\text {pulsar }}-\Delta_{\text {companion }}$ (Damour \& Schäfer 1991). The extra acceleration in this case would be in the direction of the Galactic acceleration of the system (Damour \& Schäfer 1991), accessible through potential models of the Galaxy (e.g., Kuijken \& Gilmore 1989). For the $\hat{\alpha_{1}}$ test, the direction is given by the velocity of the pulsar system relative to the Cosmic Microwave Background (Damour \& Esposito-Farèse 1992b; Bell et al. 1996). For $\hat{\alpha_{3}}$ the direction comes from the cross-product of this absolute velocity and the pulsar spin direction (Nordtvedt \& Will 1972; Bell \& Damour 1996) and the test relies on the evolutionary assumption of alignment between the pulsar spin and the orbital angular momentum. All of the tests attempt to distinguish the strength of a forced eccentricity 
component against that of the "natural" eccentricity, which should fall within a certain range based on the orbital period (Phinney 1992) and whose direction evolves according to the general-relativistic advance of periastron. The time-dependent eccentricity vector may be written as (Damour \& Schäfer 1991): $\mathbf{e}(t)=\mathbf{e}_{F}+\mathbf{e}_{R}(t)$, where $\mathbf{e}_{R}(t)$ is the rotating "natural" eccentricity vector, and $\mathbf{e}_{F}$ is the forced component.

Applying the tests requires making two important decisions. The first is the selection of pulsars. For the $\Delta_{\text {net }}$ test, the figure of merit is $P_{\mathrm{b}}^{2} / e$ (Damour \& Schäfer 1991), where $P_{\mathrm{b}}$ is the orbital period, while for $\hat{\alpha_{1}}$ it is $P_{\mathrm{b}}^{1 / 3} / e$ (Damour \& Esposito-Farèse 1992b) and for $\hat{\alpha_{3}}$ it is $P_{\mathrm{b}}^{2} /(e P)$ (Bell \& Damour 1996), where $P$ is the pulsar's spin period. This makes MSPs in long-period orbits the logical choice for $\Delta_{\text {net }}$ and $\left|\hat{\alpha}_{3}\right|$, and those in shorter-period, low-eccentricity orbits the choice for $\hat{\alpha_{1}}$. However, the pulsars must also satisfy other restrictions: they must be old enough that $\mathbf{e}_{R}(t)$ can be assumed to be randomly oriented, and have $\dot{\omega}$ (advance of periastron) larger than the rate of Galactic rotation (Damour \& Schäfer 1991; Damour \& Esposito-Farèse 1992b; Bell et al. 1996; Wex 1997). A related issue is ensuring that a set of pulsars is used representing the full related population, even those systems that might have a low figure of merit (Wex 2000). The set of pulsars to be used is reasonably clear for $\Delta_{\text {net }}$ and $\hat{\alpha_{3}}$, for which the optimal pulsars have likely all followed the same evolutionary path (e.g. Rappaport et al. 1995), but the situation is less clear for the shorter-orbital period pulsars used for $\hat{\alpha_{1}}$.

The other decision is the method for obtaining the limit. Some sampling or averaging is typically needed for the orbital inclination angle $i$, the masses $m_{1}$ and $m_{2}$, the sky orientation of the binary and the pulsar distance, though these are reasonably wellconstrained in some cases (Verbiest et al. 2008; Splaver et al. 2005). One place where progress has been made is the issue of how to treat the unknown angle between $\mathbf{e}_{\mathrm{F}}$ and the "natural" eccentricity $\mathbf{e}_{\mathrm{R}}$. Limits derived from "worst-case" cancellation scenarios have been obtained for the various tests using individual pulsars (Damour \& Schäfer 1991; Damour \& Esposito-Farèse 1992b; Bell et al. 1996) and an ensemble of pulsars (Bell \& Damour 1996; Wex 1997, 2000); Wex (2000) attempted to account for population selection effects by using a larger set of pulsars as discussed above. An alternative analysis uses a Bayesian formalism and more of the known pulsar parameters such as the longitude of periastron, again operating on the full set of pulsars that likely have similar evolutionary histories, to obtain a $95 \%$ confidence upper limit on $|\Delta|$ of $5.6 \times 10^{-3}$ and on $\left|\hat{\alpha_{3}}\right|$ of $4 \times 10^{-20}$. (Stairs et al. 2005). These limits will soon be updated with the inclusion of new pulsars, improved timing parameters for known pulsars and some small error corrections (Gonzalez et al., in prep.). For $\left|\hat{\alpha_{1}}\right|$, the most recent limit is $1.4 \times 10^{-4}$ at 95\% confidence (Wex 2000). This test could be updated with the Bayesian analysis, but the selection of pulsars is less straightforward. It should be noted that isolated pulsars can also be used to set a limit on $\hat{\alpha_{3}}$ based on the average value of the observed period derivatives (Will 1993; Bell 1996; Bell \& Damour 1996).

\subsection{Orbital Decay Tests}

The difference in self-gravitation contributions to the masses of pulsars and white dwarfs makes such binary systems targets of scrutiny for anomalous orbital decay other than that due to the quadrupolar gravitational radiation emission predicted by GR. Theories that violate the SEP may predict dipolar gravitational radiation, for example. The decrease of the period of a circular orbit due to dipolar emission can be written as (Will 1993; Damour \& Esposito-Farese 1996):

$$
\dot{P}_{\mathrm{b} \text { Dipole }}=-\frac{4 \pi^{2} G_{*}}{c^{3} P_{\mathrm{b}}} \frac{m_{1} m_{2}}{m_{1}+m_{2}}\left(\alpha_{c_{1}}-\alpha_{c_{2}}\right)^{2},
$$


where $G_{*}=G$ in GR, and $\alpha_{c_{i}}$ is the coupling strength of object " $\imath$ " to a scalar gravitational field (Damour \& Esposito-Farese 1996). In a complementary fashion, these observations may also be used to set limits on variations of Newton's constant, which would affect the binding energies of the stars as well as the angular momentum of the system. The expected orbital period derivative may be written as (Damour et al. 1988; Nordtvedt 1990):

$$
\left(\frac{\dot{P}_{\mathrm{b}}}{P_{\mathrm{b}}}\right)_{\dot{G}}=-\left[2-\left(\frac{m_{1} c_{1}+m_{2} c_{2}}{m_{1}+m_{2}}\right)-\frac{3}{2}\left(\frac{m_{1} c_{2}+m_{2} c_{1}}{m_{1}+m_{2}}\right)\right] \frac{\dot{G}}{G} .
$$

The challenge is to quantify the various possible contributions to a measured or constrained orbital period derivative (e.g., Lazaridis et al. 2009). The quadrupolar prediction is expected but may be too small to be measurable. There may be terms corresponding to mass loss or other binary evolution effects in the system. The dipolar and $\dot{G}$ contributions can be estimated with the compactness or coupling strengths remaining as parameters. Another expected contribution to any observed value is the "Shklovskii effect" (Shklovskii 1970) due to the pulsar's transverse velocity: $\dot{P}_{\mathrm{pm}}=P \mu^{2} d / c$, where $\mu$ is the proper motion and $d$ is the distance to the pulsar. This term can be difficult to estimate well in the absence of a good distance measurement. A similar term comes from the differential acceleration of the solar system and the pulsar in the gravitational field of the Galaxy (Damour \& Taylor 1991; Nice \& Taylor 1995); its determination also requires good knowledge of the pulsar's spatial position.

Not surprisingly, then, the best tests come from pulsar-white-dwarf binaries with shortperiod orbits and reliable distance estimates, often through parallax. The best dipolar radiation limit in a recycled-pulsar system comes from PSR J1012+5307, which sets a $95 \%$ confidence limit of $\left(\alpha_{c_{1}}-\alpha_{c_{2}}\right)^{2}=(0.5 \pm 6.0) \times 10^{-5}$ (Lazaridis et al. 2009). For $\dot{G} / G$, there are several similar limits: PSR J1713+0747 yields a $95 \%$ confidence limit of $\dot{G} / G=(1.5 \pm 3.8) \times 10^{-12} \mathrm{yr}^{-1}$ (Splaver et al. 2005 ; Nice et al. 2005 , D. Nice, private communication), while Deller et al. (2008) report a $95 \%$ upper limit of $(0.5 \pm 2.6) \times$ $10^{-12} \mathrm{yr}^{-1}$ using the Damour et al. (1988) expression for $\dot{G} / G$ and the pulsar J0437-4715 with slightly different error estimation. Recently, that result has been combined with timing of PSR J1012+5307 to yield a combined limit on $\dot{G} / G$ and dipolar radiation that does not need external input such as LLR limits (Lazaridis et al. 2009).

An especially interesting case is the young-pulsar-white-dwarf system PSR J1141-6545 (Kaspi et al. 2000), which is eccentric and should therefore be a strong emitter of gravitational radiation and and an excellent constrainer of dipolar gravational radiation (Gérard \& Wiaux 2002; Esposito-Farese 2005). The orbital period derivative is well-measured (Bhat et al. 2008) and agrees with the GR predictions to within 6\%, setting a limit on $\left(\alpha_{c_{1}}-\alpha_{c_{2}}\right)^{2} \simeq \alpha_{0}^{2}<3.4 \times 10^{-6}$ in the case of strongly non-linear coupling of the pulsar to the scalar field and $\alpha_{0}^{2}<2.1 \times 10^{-5}$ in the case of weakly non-linear coupling (Damour \& Esposito-Farèse 1992a; Esposito-Farese 2005; Bhat et al. 2008).

While double-neutron-star (DNS) systems can also limit dipolar gravitational radiation (e.g., Will 1977; Will \& Zaglauer 1989), this is of less interest because of their symmetry. However, their observed decrease in orbital periods can rule out those theories that predict an increase (e.g., Rosen 1973; Ni 1973; Lightman \& Lee 1973; Weisberg \& Taylor 1981). A different pulsar-derived limit to $G$ variation uses pulsar spin-down as a limit on changes due to the gravitational binding of the neutron star (Counselman \& Shapiro 1968; Goldman 1990), with MSPs being especially suitable for this task and yielding limits on $\dot{G} / G$ of a few $10^{-11} \mathrm{yr}^{-1}$ (e.g. Arzoumanian 1995). In the past, a limit also could be derived based on similarities in pulsar masses in old globular clusters and younger galactic field systems (Thorsett 1996), but the recent profusion of pulsars in globular 
clusters, some with possibly quite large mass estimates (e.g. Ransom et al. 2005; Freire et al. 2008), throws the validity of this test into question.

\section{Highly Relativistic Systems}

\subsection{Self-consistency Tests}

Measurement of orbital period derivatives is also a component of the best-known realization of binary pulsar tests of GR: verification of self-consistency between relativistic observables in DNS and similar systems. This is achieved by measuring the relativistic corrections to the Keplerian orbit (i.e., the "post-Keplerian" or "PK" parameters, which besides $\dot{P}_{\mathrm{b}}$ and $\dot{\omega}$ include the time-dilation/gravitational-redshift term $\gamma$ and the $r$ and $s$ Shapiro delay parameters) in a manner that does not assume that any particular theory of gravity is correct (Damour \& Deruelle 1985). For any theory of gravity (e.g., GR, or a tensor-scalar theory with its parameters fixed), these PK parameters will be functions only of the two stellar masses, which are the two unknowns in any single-lined spectroscopic Keplerian orbit. Thus measurement of two PK parameters will yield the masses, and measurement of three or more will result in an over-constrained system that may be checked for internal consistency. In GR, the equations describing the PK parameters in terms of the stellar masses are (Damour \& Deruelle 1986; Taylor \& Weisberg 1989; Damour \& Taylor 1992):

$$
\begin{gathered}
\dot{\omega}=3\left(\frac{P_{b}}{2 \pi}\right)^{-5 / 3}\left(T_{\odot} M\right)^{2 / 3}\left(1-e^{2}\right)^{-1}, \\
\gamma=e\left(\frac{P_{b}}{2 \pi}\right)^{1 / 3} T_{\odot}^{2 / 3} M^{-4 / 3} m_{2}\left(m_{1}+2 m_{2}\right), \\
\dot{P}_{b}=-\frac{192 \pi}{5}\left(\frac{P_{b}}{2 \pi}\right)^{-5 / 3}\left(1+\frac{73}{24} e^{2}+\frac{37}{96} e^{4}\right)\left(1-e^{2}\right)^{-7 / 2} T_{\odot}^{5 / 3} m_{1} m_{2} M^{-1 / 3}, \\
r=T_{\odot} m_{2}, \\
s=x\left(\frac{P_{b}}{2 \pi}\right)^{-2 / 3} T_{\odot}^{-1 / 3} M^{2 / 3} m_{2}^{-1} .
\end{gathered}
$$

where $x$ is the projected semi-major axis of the orbit of $m_{1}$ in light-seconds, $s \equiv \sin i, M=$ $m_{1}+m_{2}$ (all masses in units of solar mass), and $T_{\odot} \equiv G M_{\odot} / c^{3}=4.925490947 \mu \mathrm{s}$. Other theories of gravity will have somewhat different mass dependencies for these parameters.

The self-consistency test is often illustrated by a "mass-mass diagram" such as the one for the double pulsar J0737-3039A/B in GR in Figure 1. When the curves intersect in a common region, as they do in this case, the parameters are said to agree with the theory being tested. This type of test was pioneered for the first DNS PSR B1913+16 (Hulse \& Taylor 1975; Taylor \& Weisberg 1982, 1989) and has now also been accomplished for PSRs B1534+12 (Stairs et al. 2002), the double pulsar (Kramer et al. 2006), J1756-2251 (to low precision; Ferdman 2008) and the white-dwarf binary J1141-6545 (Bhat et al. 2008). Of these, B1534+12, J0737-3039A/B and (again to low precision) J1756-2251 allow tests that do not include the orbital period decay and hence provide an important "quasi-static" complement to the Hulse-Taylor case (Taylor et al. 1992). Combination of results from all the pulsars has potential to set significant constraints on deviations from GR (e.g., Taylor et al. 1992; Esposito-Farese 2005). Such systems can also be used to limit preferred-frame effects in a novel way (Wex \& Kramer 2007). 


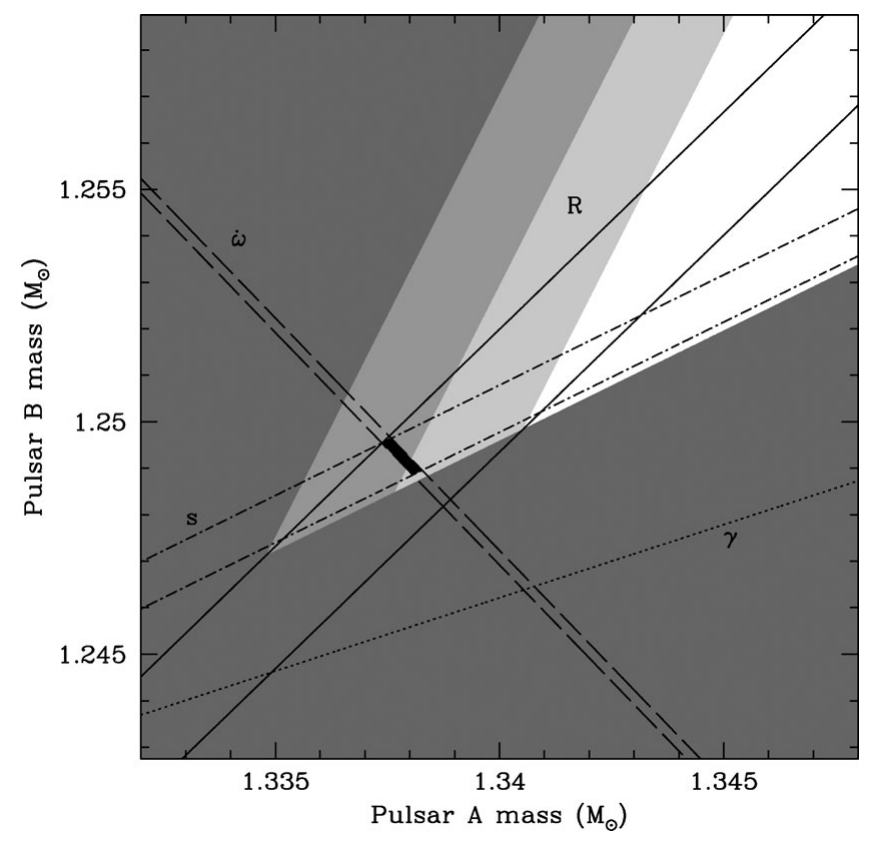

Figure 1. A section of the mass-mass diagram for the double pulsar J0737-3039A/B within GR, based on the measurement of 5 PK parameters in the recycled "A" pulsar and the mass ratio $R$, which is derived from the relative sizes of the "A" and "B" orbits (Kramer et al. 2006). Only the lower curve for $\gamma$ falls within this plot. The grey-shaded regions are those forbidden by the mass functions of the two pulsars, with the deepening shades of grey illustrating the sizeable uncertainty in the mass function for B. The intersection of all the curves in one area (shaded black) indicates that GR passes the self-consistency test in this system. Note that 1) the similar plot in Stairs (2008) mistakenly used incorrect uncertainties for $x_{B}$ and for $s$ and 2) the $s$ curves plotted here correspond directly to the asymmetric $68 \%$ range in $s$ listed in Kramer et al. (2006), slightly different from the plotting approximation used in that paper.

The double pulsar has another crucial advantage in the measurement of the mass ratio $R$, which has the same dependence on the masses in all gravitational theories, at least to order $(v / c)^{2}$ (Damour \& Taylor 1992). For this system, the combination of $R$ and $\dot{\omega}$ allows an estimate of the masses and prediction of all the other PK parameters, with the Shapiro delay $s$ parameter agreeing with the prediction to within $0.05 \%$, the most stringent test to date of strong-field gravity (Kramer et al. 2006). In future, this system may allow measurement of high-order corrections to $\dot{\omega}$, such as that due to spin-orbit coupling of the recycled "A" pulsar; in turn this may provide a constraint on the NS equation of state (e.g,. Lattimer \& Schutz 2005).

\subsection{Geodetic Precession}

As discussed above, the supernova explosion that creates a DNS (or similar) system is expected to leave the spin axes of the stars misaligned with the orbital angular momentum. This will result in geodetic precession of the NS spins about the total angular momentum (dominated by the orbital component) (Damour \& Ruffini 1974; Barker \& O'Connell 1975). Example precession periods are roughly 700 years for PSR B1534+12, and 75 and 71 years for PSR J0737-3039A and B, respectively.

One means of identifying this effect is through secular changes in the observed pulse profiles, as different parts of the emission region come into view. Such changes were soon noticed in the Hulse-Taylor binary (Weisberg et al. 1989): a difference in the relative 


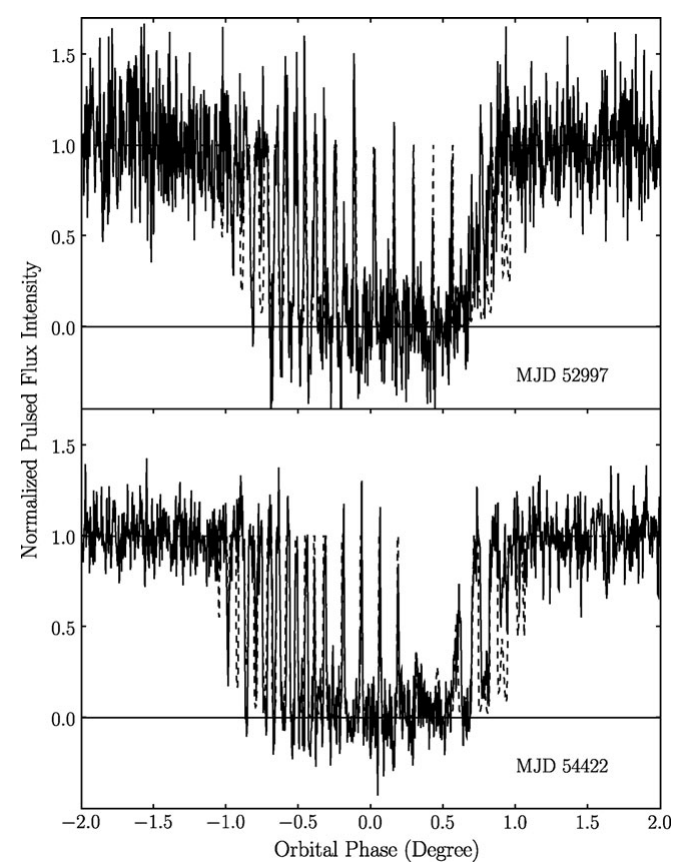

Figure 2. Changing eclipses in the double pulsar. The plots show the relative flux density of the A pulsar as a function of orbital phase at two different epochs (solid lines) as well as fits to the Lyutikov (2005) model (dashed lines). The secular changes in the short-term modulation indicate the changing precession phase of B's spin axis. Figure courtesy of René Breton.

heights of the two profile peaks over several years. In the 1990s, the peaks began to draw together, suggesting a spin-orbit misalignment angle of about $22^{\circ}$ and allowing a prediction that the pulsar will disappear from view in about 2025 (Kramer 1998). Profile shape changes as well as polarization changes are seen in PSR B1534+12 (Arzoumanian 1995; Stairs et al. 2004); the profile changes are also reflected in orbital-timescale aberrational variations, allowing a beam-model-independent, though low-precision, test of the precession rate, which agrees with the GR prediction (Stairs et al. 2004). Geodetic precession has also been observed in the pulsar-white-dwarf system PSR J1141-6545 (Hotan et al. 2005, Manchester et al., in prep.) and there is good evidence for the effect in the recently discovered PSR J1906+0746 (Lorimer et al. 2006, Kasian et al. 2008).

Once again, the double pulsar provides a unique and fascinating view on the problem. Profile shape changes are seen in the young "B" pulsar (Burgay et al. 2005), which also shows large orbitally-dependent profile variations (Lyne et al. 2004). The recycled "A" pulsar shows no changes, however, which may have interesting implications for the "B" supernova (Manchester et al. 2005; Ferdman 2008; Ferdman et al. 2008, and references therein). But the combination of the two pulsars allows an even more powerful probe. Because the orbit is nearly edge-on to the line of sight, the A pulsar is eclipsed by the magnetosphere of B for about 30 seconds per orbit (Lyne et al. 2004; Kaspi et al. 2004; Lyutikov \& Thompson 2005), with the flux of A showing strong modulation at the spin period of B and its second harmonic (McLaughlin et al. 2004). The overall behaviour is nicely described by a simple dipolar field structure for the B pulsar with a well-defined orientation (Lyutikov 2005). Changes in the A flux modulation pattern over time (see Figure 2) can be matched up to changes in the B spin orientation, resulting in a $13 \%$ measurement of B's spin-orbit precession rate that agrees beautifully with the GR 
prediction (Breton et al. 2008). Moreover, because the sizes of both orbits are known, this test in fact applies to a large generic class of fully conservative, Lagrangian-based gravitational theories (Damour \& Taylor 1992; Breton et al. 2008).

The planned Square Kilometre Array will vastly improve all of these tests, and furthermore has great potential for finding highly exotic systems such as pulsar-black-hole binaries, which would open new realms of gravitational exploration (Kramer et al. 2004).

\section{Acknowledgements}

Pulsar research at UBC is supported by an NSERC Discovery Grant. The author also acknowledges sabbatical support from the Swinburne University of Technology Visiting Distinguished Researcher Scheme. She thanks her many collaborators on some of the results discussed here, and especially Michael Kramer for a careful reading of the manuscript and René Breton for providing Fig. 2.

\section{References}

Arzoumanian, Z. 1995, PhD thesis, Princeton University

Bailes, M. in this proceedings, 212

Barker, B. M. \& O'Connell, R. F. 1975, Phys. Rev. D, 12, 329

Bassa, C., Wang, Z., Cumming, A., \& Kaspi, V., eds. 2008, AIP Conf. Proc. 983: 40 Years of Pulsars (Springer)

Bell, J. F. 1996, ApJ, 462, 287

Bell, J. F., Camilo, F., \& Damour, T. 1996, ApJ, 464, 857

Bell, J. F. \& Damour, T. 1996, Class. Quant Grav., 13, 3121

Bhat, N. D. R., Bailes, M., \& Verbiest, J. P. W. 2008, Phys. Rev. D, 77, 124017

Breton, R. P. et al. 2008, Science, 321, 104

Burgay, M. et al. 2005, ApJ, 624, L113

Counselman, C. C. \& Shapiro, I. I. 1968, Science, 162, 352

Damour, T. \& Deruelle, N. 1985, Ann. Inst. H. Poincaré (Physique Théorique), 43, 107

—. 1986, Ann. Inst. H. Poincaré (Physique Théorique), 44, 263

Damour, T. \& Esposito-Farèse, G. 1996, Phys. Rev. D, 53, 5541

Damour, T. \& Esposito-Farèse, G. 1992a, Class. Quant Grav., 9, 2093

-. 1992b, Phys. Rev. D, 46, 4128

Damour, T. \& Esposito-Farese, G. 1996, Phys. Rev. D, 54, 1474

Damour, T., Gibbons, G. W., \& Taylor, J. H. 1988, Phys. Rev. Lett., 61, 1151

Damour, T. \& Ruffini, R. 1974, Academie des Sciences Paris Comptes Rendus Ser. Scie. Math., 279,971

Damour, T. \& Schäfer, G. 1991, Phys. Rev. Lett., 66, 2549

Damour, T. \& Taylor, J. H. 1991, ApJ, 366, 501

-. 1992, Phys. Rev. D, 45, 1840

Deller, A. T., Verbiest, J. P. W., Tingay, S. J., \& Bailes, M. 2008, ApJ, 685, L67

Eardley, D. M. 1975, ApJ, 196, L59

Esposito-Farese, G. 2005, in The Tenth Marcel Grossmann Meeting, ed. M. Novello, S. Perez Bergliaffa, \& R. Ruffini (World Scientific Publishing), 647

Ferdman, R. D. 2008, PhD thesis, University of British Columbia

Ferdman, R. D. et al. 2008, in Bassa et al. (2008), 474

Freire, P. C. C., et al. 2008, ApJ, 675, 670

Gérard, J.-M. \& Wiaux, Y. 2002, Phys. Rev. D, 66, 1

Goldman, I. 1990, MNRAS, 244, 184

Hotan, A. W., Bailes, M., \& Ord, S. M. 2005, ApJ, 624, 906

Hulse, R. A. \& Taylor, J. H. 1975, ApJ, 195, L51

Kasian, L. \& the PALFA Consortium. 2008, in in Bassa et al. (2008), 485

Kaspi, V. M. et al. 2000, ApJ, 543, 321 
Kaspi, V. M. et al. 2004, ApJ, 613, L137

Kramer, M. 1998, ApJ, 509, 856

Kramer, M. et al. 2004, New Astr., 48, 993

Kramer, M. et al. 2006, Science, 314, 97

Kuijken, K. \& Gilmore, G. 1989, MNRAS, 239, 571

Lattimer, J. M. \& Schutz, B. F. 2005, ApJ, 629, 979

Lazaridis, K. et al. 2009, MNRAS, in press, arXiv:0908.0285

Lightman, A. \& Lee, D. 1973, Phys. Rev. D, 8, 3293

Lorimer, D. R. \& Kramer, M. 2005, Handbook of Pulsar Astronomy (Cambridge University Press)

Lorimer, D. R. et al. 2006, ApJ, 640, 428

Lyne, A. G. et al. 2004, Science, 303, 1153

Lyutikov, M. 2005, MNRAS, 362, 1078

Lyutikov, M. \& Thompson, C. 2005, ApJ, 634, 1223

Manchester, R. N. et al. 2005, ApJ, 621, L49

McLaughlin, M. A. et al., 2004, ApJ, 616, L131

Ni, W. 1973, Phys. Rev. D, 7, 2880

Nice, D. J. et al. 2005, ApJ, 634, 1242

Nice, D. J. \& Taylor, J. H. 1995, ApJ, 441, 429

Nordtvedt, K. 1968, Phys. Rev., 170, 1186

—. 1990, Phys. Rev. Lett., 65, 953

Nordtvedt, K. \& Will, C. M. 1972, ApJ, 177, 775

Phinney, E. S. 1992, Phil. Trans. Roy. Soc. A, 341, 39

Ransom, S. M. et al. 2005, Science, 307, 892

Rappaport, S. et al. 1995, MNRAS, 273, 731

Rosen, N. 1973, Gen. Relativ. Gravit., 4, 435

Shklovskii, I. S. 1970, Sov. Astron., 13, 562

Splaver, E. M., Nice, D. J., Stairs, I. H., Lommen, A. N., \& Backer, D. C. 2005, ApJ, 620, 405

Stairs, I. H. 2003, Living Reviews in Relativity, 5, uRL (Cited on 2008/02/16): http://relativity.livingreviews.org/Articles/lrr-2003-5

Stairs, I. H. 2005, in Binary Radio Pulsars, ed. F. Rasio \& I. H. Stairs (San Francisco: Astronomical Society of the Pacific), 3

Stairs, I. H. 2006, in Proceedings of 33rd SLAC Summer Institute on Particle Physics (SSI 2005): Gravity in the Quantum World and the Cosmos, 2005, Vol. eConf C0507252, L004

Stairs, I. H. 2008, in Bassa et al. (2008), 424

Stairs, I. H. et al. 2005, ApJ, 632, 1060

Stairs, I. H., Thorsett, S. E., \& Arzoumanian, Z. 2004, Phys. Rev. Lett., 93, 141101

Stairs, I. H., Thorsett, S. E., Taylor, J. H., \& Wolszczan, A. 2002, ApJ, 581, 501

Tauris, T. M. \& van den Heuvel, E. P. J. 2006, Formation and Evolution of Compact Stellar X-ray Sources, ed. W. H. G. Lewin \& M. van der Klis (Cambridge: Cambridge University Press), 623-665

Taylor, J. H. \& Weisberg, J. M. 1982, ApJ, 253, 908

-. 1989, ApJ, 345, 434

Taylor, J. H., Wolszczan, A., Damour, T., \& Weisberg, J. M. 1992, Nature, 355, 132

Thorsett, S. E. 1996, Phys. Rev. Lett., 77, 1432

Verbiest, J. P. W. et al. 2008, ApJ, 679, 675

Wagoner, R. V. 1975, ApJ, 196, L63

Weisberg, J. \& Taylor, J. 1981, Gen. Relativ. Gravit., 13, 1

Weisberg, J. M., Romani, R. W., \& Taylor, J. H. 1989, ApJ, 347, 1030

Wex, N. 1997, A\&A, 317, 976

Wex, N. 2000, in Pulsar Astronomy - 2000 and Beyond, IAU Colloquium 177, ed. M. Kramer, N. Wex, \& R. Wielebinski (San Francisco: Astronomical Society of the Pacific), 113

Wex, N., Kalogera, V., \& Kramer, M. 2000, ApJ, 528, 401

Wex, N. \& Kramer, M. 2007, MNRAS, 380, 455 
Will, C. 2006, Living Reviews in Relativity, 9, 1, uRL (Cited on 2008/02/16): http://relativity.livingreviews.org/Articles/lrr-2006-3

Will, C. M. 1977, ApJ, 214, 826

-. 1993, Theory and Experiment in Gravitational Physics (Cambridge: Cambridge University Press)

Will, C. M. \& Nordtvedt, K. J. 1972, ApJ, 177, 757

Will, C. M. \& Zaglauer, H. W. 1989, ApJ, 346, 366

Williams, J. G., Turyshev, S. G., \& Boggs, D. H. 2009, International Journal of Modern Physics D, 18,1129 